PROCEEDINGS OF THE

AMERICAN MATHEMATICAL SOCIETY

Volume 137, Number 12, December 2009, Pages 4203-4207

S 0002-9939(09)10057-6

Article electronically published on August 5, 2009

\title{
A PROOF OF BOCHNER'S TUBE THEOREM
}

\author{
J. HOUNIE
}

(Communicated by Franc Forstneric)

\begin{abstract}
We give a proof of Bochner's tube theorem based on the BaouendiTreves approximation formula.
\end{abstract}

\section{INTRODUCTION}

The classical Bochner tube theorem $([\overline{\mathrm{Bo}}, \overline{\mathrm{BM}}])$ states that if $\omega \subset \mathbb{R}^{m}$ is a connected open set, any holomorphic function $f(z)$ defined on the tube $\Omega=T(\omega) \doteq$ $\omega+i \mathbb{R}^{m} \subset \mathbb{C}^{m}$ can be holomorphically extended to the convex hull $\operatorname{ch}(\Omega)$. This theorem has been extended in several directions by different authors ( $\mathrm{Ko}, \mathrm{Ka}$, [BD]). A standard ingredient in the proof of the classical result, as well as in many generalizations, is some version of what is known as the lemma of the folding screen. See, e.g., [H1, Lemma 2.5.11], [Ka, Lemma 4.1], [Ka, Prop 5.1], BD, Lemma 3.2], [Ko]. The simplest version of the folding screen lemma roughly says that if $\omega \subset \mathbb{R}^{2}$ contains the set

$$
K=\left\{\left(x_{1}, 0\right): \quad 0 \leq x_{1} \leq 1\right\} \cup\left\{\left(0, x_{2}\right): \quad 0 \leq x_{2} \leq 1\right\},
$$

then the $\Omega$-hull of $K+i\left\{y_{1}^{2}+y_{2}^{2}<r^{2}\right\}$ must contain a set $T\left(K_{r}\right)=K_{r}+i \mathbb{R}^{2}$, with $K \subset K_{r} \subset \operatorname{ch}(K)$ with the property that, as $r \nearrow \infty$, the sets $K_{r}$ fill up $\operatorname{ch}(K)$. Roughly speaking, the classical proof goes as follows. Given a holomorphic function $f(z)$ defined on the tube $\Omega=T(\omega)$, by considering sets like $T(K)$ contained in $T(\omega)$, looking at the Taylor series of $f(z)$ centered at points in $T(K)$ and invoking the folding screen lemma, one may continue analytically $f(z)$ beyond each $T(K)$. Then, to address monodromy difficulties, a delicate and indirect argument shows, assuming that $\omega$ is star-shaped, that analytic continuations corresponding to different Taylor series agree. Then the general case is proved (see [H1, p. 42] for details).

The idea behind the proof we offer here is to show that

(a) there is a sequence of entire functions $f_{j}(z)$ converging to $f(z)$ uniformly over compact subset of sets $\Omega$; and

(b) $\operatorname{ch}(\Omega)$ is contained in the domain of convegence of the sequence $\left(f_{j}\right)$, obtaining the extension in a simple way.

Received by the editors April 13, 2009

2000 Mathematics Subject Classification. Primary 32A07; Secondary 32D10, 32D15.

Key words and phrases. Bochner's tube theorem, Baouendi-Treves approximation formula.

This work was supported in part by CNPq and FAPESP.

(C)2009 American Mathematical Society
Reverts to public domain 28 years from publication 
The approximating sequence is obtained by an appropriate version of the BaouendiTreves approximation formula [BT] which furnishes a more tangible expression for the extension of $f$ as a limit of explicit integral formulas that depend on the restriction of $f$ to a totally real submanifold of the tube. The convergence of the sequence on $\operatorname{ch}(\Omega)$ follows from constructing appropriate analytic discs, which is quite simple in the tube situation.

\section{Definitions And lemmas}

Given a set $X \subset \mathbb{R}^{m}$, the tube $T(X)$ over $X$ is the subset of $\mathbb{C}^{m}$ defined by

$$
T(X)=X+i \mathbb{R}^{m}=\left\{z=x+i y: \quad x \in X, y \in \mathbb{R}^{m}\right\} .
$$

Let us denote the unit disc in $\mathbb{C}$ by $\Delta=\{z \in \mathbb{C}:|z|<1\}$ and by $\bar{\Delta}$ its closure. A continuous function $A: \bar{\Delta} \rightarrow \mathbb{C}^{m}$ is called an analytic disc in $\mathbb{C}^{m}$ if it is holomorphic on $\Delta$. We say that the point $A(0)$ is the center of the analytic disc and the subset $A(\partial \Delta)$ is the boundary of the analytic disc. If $X$ is a subset of $\mathbb{R}^{m}$ or $\mathbb{C}^{m}$ we denote by $\operatorname{ch}(X)$ the convex hull of $X$, i.e., the set of linear convex combinations $\left\{\sum_{j=1}^{N} t_{j} x_{j}, x_{j} \in X, \sum_{j=1}^{N} t_{j}=1, t_{j} \geq 0\right\}$.

Lemma 1.1. Let $\omega \subset \mathbb{R}^{m}$ be an open connected set and assume that $x_{0}, x_{1} \in \omega$, $\eta \in \mathbb{R}^{m}$. For any $0<t<1$ there exists an analytic disc

$$
A: \bar{\Delta} \rightarrow \mathbb{C}^{m}
$$

such that

(1) $A(\partial \Delta) \subset \Omega$,

(2) $A(0)=t x_{0}+(1-t) x_{1}+i \eta \doteq x_{t}+i \eta$.

Lemma 1.2. Let $\omega \subset \mathbb{R}^{m}$ be an open and connected set and let $f(z)$ be a holomorphic function defined on the tube $\Omega=T(\omega)$. There exists a sequence of entire holomorphic functions $f_{j}(z), j \in \mathbb{N}$, that converges to $f(z)$ uniformly over compact subsets of $\Omega$.

These two lemmas easily imply

Theorem 1.1 (Bochner's tube theorem). Let $\omega \subset \mathbb{R}^{m}$ be an open connected set. Every holomorphic function $f(z)$ defined on the tube $\Omega=T(\omega)$ can be extended as a holomorphic function defined on $\operatorname{ch}(\Omega)=T(\operatorname{ch}(\omega))$.

Proof. Consider the open set $\omega_{1} \subset \operatorname{ch}(\omega)$ of convex combinations with two terms $t x_{1}+(1-t) x_{2}, x_{1}, x_{2} \in \omega, 0 \leq t \leq 1$, then the set $\omega_{2}$ of points of the form $t x_{1}+(1-t) x_{2}, x_{1}, x_{2} \in \omega_{1}, 0 \leq t \leq 1$, and so on. In this way we obtain an increasing sequence of open sets $\omega \subset \omega_{1} \subset \omega_{2} \subset \cdots \subset \operatorname{ch}(\omega)$ that becomes stable after a finite number of steps. In fact, by Carathéodory's theorem [H2, p. 41], $\omega_{m}=\operatorname{ch}(\omega)$. We will show how to extend $f(z)$ from $T(\omega)$ to $T\left(\omega_{1}\right)$, then the same procedure will allow us to extend $f(z)$ from $T\left(\omega_{1}\right)$ to $T\left(\omega_{2}\right)$ and after $m$ iterations the extension to $\omega_{m}=\operatorname{ch}(\omega)$ will be reached.

Consider the sequence $f_{j}(z)$ given by Lemma 1.2. To extend $f(z)$ to $T\left(\omega_{1}\right)$ it is enough to show that every point of $T\left(\omega_{1}\right)$ has a neighborhood on which the sequence $f_{j}(z)$ converges uniformly, since in this case the limit will define a holomorphic function on $T\left(\omega_{1}\right)$ that extends $f$. Applying Lemma 1.1 to a generic point $z_{t}=$ $x_{t}+i \eta \in T\left(\omega_{1}\right), x_{0}, x_{1} \in \omega, \eta \in \mathbb{R}^{m}$, we obtain an analytic disc centered at $z_{t}$ and boundary contained in a compact subset of $\Omega$. By the maximum principle $f_{j} \circ A$ is 
a Cauchy sequence at the origin because $f_{j}(z)$ converges uniformly on the compact set $A(\partial \Delta)$. Then, by taking small translations of $A$, we obtain a family of analytic $\operatorname{discs} A_{\zeta}=\zeta+A, \zeta \in \mathbb{C}^{m},|\zeta|<\varepsilon$, whose centers cover a neighborhood of $z_{t}$ and whose boundaries are contained in a fixed compact subset of $\Omega$. Hence, $f_{j}(z)$ converges uniformly in a neighborhood of $z_{t}$, as we wished to prove.

\section{Proofs of the Lemmas}

2.1. Proof of Lemma 1.1. We may take coordinates in $\mathbb{C}^{m}$ such that $x_{0}=0$, $x_{1}=(1,0, \ldots, 0)$, and $\eta=0$. By connectedness, there exists a smooth curve $\gamma(s):[0,1] \rightarrow \omega$ such that $\gamma(0)=0$ and $\gamma(1)=x_{1}$, and we may assume that $\gamma(s)$ is flat at $s=0$ and $s=1$, i.e., all derivatives of positive order $\gamma^{(k)}(s), k \geq 1$, vanish at the endpoints $s=0$ and $s=1$. Choose $0<\varepsilon<\min (t, 2 \pi(1-t))$ and define a function $u: \partial \Delta \rightarrow \mathbb{R}^{m}$ as follows

$$
u\left(e^{i \theta}\right)= \begin{cases}0 & \text { if } 0 \leq \theta \leq 2 \pi t-\varepsilon \\ \gamma\left(\frac{\theta-2 \pi t+\varepsilon}{\varepsilon}\right) & \text { if } 2 \pi t-\varepsilon \leq \theta \leq 2 \pi t \\ x_{1} & \text { if } 2 \pi t \leq \theta \leq 2 \pi-\varepsilon \\ \gamma\left(\frac{2 \pi-\theta}{\varepsilon}\right) & \text { if } 2 \pi-\varepsilon \leq \theta \leq 2 \pi\end{cases}
$$

Set

$$
K(w)=\frac{1}{2 \pi} \int_{0}^{2 \pi} \frac{e^{i \theta}+w}{e^{i \theta}-w} u\left(e^{i \theta}\right) d \theta, \quad|w|<1, \quad 0<t<1 .
$$

Notice that, since $u\left(e^{i \theta}\right)$ is smooth, $K(w)$ can be smoothly extended up to $|w|=1$ and

$$
K\left(e^{i \theta}\right)=u\left(e^{i \theta}\right)+i H u\left(e^{i \theta}\right),
$$

where $H$ denotes the Hilbert transform. Then $K$ is an analytic disc with boundary contained in $\Omega$. The center of $K$ is

$$
\begin{aligned}
K(0) & =\frac{1}{2 \pi} \int_{0}^{2 \pi} u\left(e^{i \theta}\right) d \theta \\
& =\frac{1}{2 \pi} \int_{2 \pi t}^{2 \pi-\varepsilon} x_{1} d \theta+O(\varepsilon) \\
& =(1-t) x_{1}+O(\varepsilon)=x_{t}+O(\varepsilon) .
\end{aligned}
$$

By taking $\varepsilon>0$ small, we may assume that the error term is smaller than the distance $\delta=\operatorname{dist}(\gamma([0,1]), \partial \Omega)$ from $\gamma([0,1])$ to $\partial \Omega$. Hence, we may find $\zeta \in \mathbb{C}^{m}$, $|\zeta|<\delta$, such that the translated analytic disc $A(w)=\zeta+K(w)$ has the required properties, namely, $A(\partial \Delta) \subset \Omega$ and $A(0)=x_{t}$.

The next lemma, the proof of which we include for the sake of completeness, is based on a standard variation of a particular case of the Baouendi-Treves approximation theorem.

2.2. Proof of Lemma 1.2. We will assume, without loss of generality, that $0 \in \omega$. Let $f(z)=f(x+i y)$ be a holomorphic function defined on $\Omega=T(\omega)=\omega+i \mathbb{R}^{m}$. Choose a function $h(y) \in C_{c}^{\infty}\left(\mathbb{R}^{m}\right)$ satisfying $h(y)=0$ for $|y| \geq 2, h(y)=1$ for $|y| \leq 1$ and set $h_{r}(y) \doteq h(y / r)$. For $\tau, r>0$, the approximation operator is

$$
E_{\tau, r} f(z)=(\tau / \pi)^{m / 2} \int_{\mathbb{R}^{m}} e^{\tau[z-i \eta]^{2}} f(0+i \eta) h_{r}(\eta) d \eta, \quad z \in \mathbb{C}^{m} .
$$


Here $[\zeta]^{2} \doteq \zeta_{1}^{2}+\cdots+\zeta_{m}^{2}$ for $\zeta=\left(\zeta_{1}, \ldots, \zeta_{m}\right) \in \mathbb{C}^{m}$, which explains the meaning of $[z-i \eta]^{2}$ in the formula. Clearly, $E_{\tau, r} f(z)$ is an entire function of $z$. The modified approximation operator is

$$
\begin{gathered}
G_{\tau, r} f(x, y)=(\tau / \pi)^{m / 2} \int_{\mathbb{R}^{m}} e^{-\tau|y-\eta|^{2}} f(x+i \eta) h_{r}(\eta) d \eta, \\
(x, y) \in \omega \times \mathbb{R}^{m},
\end{gathered}
$$

and the remainder operator is

$$
G_{\tau, r} f(x, y)-E_{\tau, r} f(x+i y)=R_{\tau, r} f(x, y), \quad x+i y \in \Omega .
$$

If $x$ belongs to a ball of $\mathbb{R}^{m}$ centered at the origin and contained in $\omega$, a convenient formula for the remainder operator expresses it as a path integral of a one-form (see [BCH, p. 64])

$$
R_{\tau, r} f(x, y)=\int_{[0, x]} \sum_{j=1}^{m} r_{j}(x, y, \xi, \tau, r) d \xi_{j},
$$

where $[0, x]$ denotes the straight segment in $\mathbb{R}^{m}$ that joins 0 to $x$ and the coefficients of the one-form are given by

$$
r_{j}(x, y, \xi, \tau, r)=(\tau / \pi)^{m / 2} \int_{\mathbb{R}^{m}} e^{\tau[x-\xi+i(y-\eta)]^{2}} f(\xi+i \eta) L_{j} h_{r}(\eta)(-i)^{m} d \zeta .
$$

Here $L_{j}$ denotes the vector field $L_{j}=-i \partial / \partial \eta_{j}$ and $d \zeta=d \zeta(\xi, \eta)$ is the $m$-form

$$
\begin{aligned}
d \zeta(\xi, \eta) & =d \zeta_{1} \wedge d \zeta_{2} \wedge \cdots \wedge d \zeta_{m} \\
& =d\left(\xi_{1}+i \eta_{1}\right) \wedge d\left(\xi_{2}+i \eta_{2}\right) \wedge \cdots \wedge d\left(\xi_{m}+i \eta_{m}\right) .
\end{aligned}
$$

More generally, if $x \in \omega$ and $\gamma_{x}$ is a polygonal path joining the origin to $x$, we still have the analogous expression

$$
R_{\tau, r} f(x, y)=\int_{\gamma_{x}} \sum_{j=1}^{m} r_{j}(x, y, \xi, \tau, r) d \xi_{j}
$$

because the proof of the formula involves integration of an exact one-form whose integral is path independent. Let $\omega_{1} \subset \omega$ be open, connected and relatively compact, let $R>0$ be a large number and denote by $B_{R} \subset \mathbb{R}^{m}$ the ball of radius $R$ centered at the origin. If $\gamma_{x} \subset \omega_{1}$, we have the estimate

$$
\begin{gathered}
\left|R_{\tau, r} f(x, y)\right| \leq C\left|\gamma_{x}\right| \max _{1 \leq j \leq n} \sup _{\xi \in \omega_{1}}\left|r_{j}(x, y, \xi, \tau, r)\right|, \\
\text { for }(x, y) \in \omega_{1} \times B_{R} .
\end{gathered}
$$

However, due to the fact that the factor $L_{j} h_{r}(\eta)$ vanishes for $|\eta| \leq r$, choosing $r>2 R$ we have

$$
\left|e^{\tau[x-\xi+i(y-\eta)]^{2}}\right| \leq e^{-c \tau}, \quad(x, y) \in \omega_{1} \times B_{R},|\eta| \geq r, \xi \in \omega_{1},
$$

for some $c>0$. Furthermore, if we take $\omega_{1}$ as the interior of a finite and connected union of closed dyadic cubes, we may find a constant $C_{\omega_{1}}$ such that all points $x \in \omega_{1}$ can be reached by a polygonal line $\gamma_{x}$ of length $\left|\gamma_{x}\right| \leq C_{\omega_{1}}$. Therefore,

$$
\left|R_{\tau, r} f(x, y)\right| \leq C e^{-c \tau}, \quad(x, y) \in \omega_{1} \times B_{R}, r>2 R,
$$

showing that $R_{\tau, r} f(x, y) \rightarrow 0$ uniformly on $\omega_{1} \times B_{R}$ for $r>2 R$. Thus, given any compact subset $K \Subset \Omega$, and $\varepsilon>0$ we may find $R>0$ and $\omega_{1}$ as above with $\omega_{1} \Subset \omega$ such that $K \subset \omega_{1} \times B_{R}$, and then choose $r>2 R$ and $\tau>0$ large enough so that 
$\left|R_{\tau, r} f(x, y)\right|<\varepsilon$ on $K$. On the other hand, the modified approximation operator (2.1) is the approximation of the identity associated to the Gaussian and converges uniformly to $f(x+i y) h_{r}(y)$ on $\omega_{1} \times \mathbb{R}^{m}$, which agrees with $f(x+i y)$ for $|y|<R$. Hence, (2.2) shows that $\left|E_{\tau, r} f(z)-f(z)\right|<2 \varepsilon$ for $z \in K$ if $r>2 R$ and $\tau$ is large enough. Using a diagonal procedure, one may now find sequences $r_{j} \nearrow \infty, \tau_{j} \nearrow \infty$, such that $f_{j}(z)=E_{\tau_{j}, r_{j}} f(z)$ converges to $f(z)$ uniformly on compact sets of $\Omega$.

\section{Final REMARKS}

A domain $\Omega \subset \mathbb{C}^{m}$ is said to be a Runge domain if it is a domain of holomorphy and every holomorphic function on $\Omega$ can be uniformly approximated over compact subsets of $\Omega$ by entire functions [H1, p. 52] (some authors [V] p. 216] do not require that $\Omega$ be a domain of holomorphy). It is well known that convex open sets are Runge domains with the more stringent definition.

A known consequence of Bochner's tube theorem is that any holomorphic function $f(z)$ defined on a tube $\Omega=T(\omega) \subset \mathbb{C}^{m}$ may be approximated by entire functions. The argument goes as follows. Start by extending $f(z)$ to $\operatorname{ch}(\Omega)$. Since the latter set is convex, therefore a Runge domain, the extension may be approximated by entire functions on $\operatorname{ch}(\Omega)$ and a fortiori on $\Omega$. In this work we have taken the opposite path, showing directly that any holomorphic function of $\Omega$ may be approximated by entire functions and then, using this fact, we prove Bochner's theorem.

\section{REFERENCES}

[BT] M. S. Baouendi and F. Treves, A property of the functions and distributions annihilated by a locally integrable system of complex vector fields, Ann. of Math. (2) 113 (1981), 387-421. MR607899 (82f:35057)

$[\mathrm{BCH}]$ S. Berhanu, P. Cordaro and J. Hounie, An Introduction to Involutive Structures, Cambridge University Press, 2008. MR2397326 (2009b:32048)

[Bo] S. Bochner, A theorem on analytic continuation of functions in several variables, Ann. of Math. (2) 39 (1938), 14-19. MR.1503384

[BM] S. Bochner and W. T. Martin, Several Complex Variables, Princeton University Press, 1948. MR0027863 (10:366a)

[BD] A. Boivin and R. Dwilewicz, Extension and approximation of CR functions on tube manifolds, Trans. Amer. Math. Soc. 350 (1998), 1945-1956. MR1443864 (98h:32011)

[H1] L. Hörmander, An Introduction to Complex Analysis in Several Variables, North-Holland, 1973. MR0344507 (49:9246)

[H2] L. Hörmander, Notions of Convexity, Birkhäuser Boston, 1994. MR.1301332 (95k:00002)

[Ka] M. Kazlow, CR functions and tube manifolds, Trans. Amer. Math. Soc. 255 (1979), 153-171. MR542875 (80m:32001)

[Ko] H. Komatsu, A local version of Bochner's tube theorem, J. Fac. Sci. Univ. Tokyo Sect. 1A Math. 19 (1972), 201-214. MR0316749 (47:5297)

[V] V. S. Vladimirov, Methods of the Theory of Functions of Many Complex Variables, The M.I.T. Press, 1966. MR0201669 (34:1551)

Departamento de Matemática, Universidade Federal de São Carlos, São Carlos, SP, 13565-905, BRASIL

E-mail address: hounie@dm.ufscar.br 